\title{
Effects of Bisoprolol and Flecainide in an Elderly Patient with Paroxysmal Supraventricular Tachycardia and Atrial Fibrillation in Wolff-Parkinson-White Syndrome

\author{
Jae-Jin Kwak*, Joon Hyung Doh and Sung Yun Lee
}

Division of Cardiology, Department of Internal Medicine and Cardiovascular Center, Inje University IIsan Paik Hospital, South Korea

*Corresponding author: Jae-Jin Kwak, Division of Cardiology, Department of Internal Medicine and Cardiovascular Center, Ilsan Paik Hospital, 170 Joohwa-ro, Ilsanseo-gu, Goyang-si, Gyeonggi-do 10380, South Korea, Tel : +82-31-9107830, Fax : +82-31-910-7829

\begin{abstract}
Wolff-Parkinson-White (WPW) syndrome can develop paroxysmal supraventricular tachycardia (PSVT) or/and paroxysmal atrial fibrillation (AF). AF in WPW syndrome is a potentially life-threatening arrhythmia. Radiofrequency catheter ablation is recommended in patients with $A F$ in WPW syndrome. Atrioventricular nodal blockers can precipitate ventricular fibrillation and should not be used. We present patients with PSVT and AF in WPW syndrome who was treated with bisoprolol and flecainide.
\end{abstract}

\section{Keywords}

Wolff-Parkinson-White syndrome, Tachycardia, Atrial fibrillation, Flecainide, Bisoprolol

\section{Introduction}

Wolff-Parkinson-White (WPW) syndrome is the most common form of ventricular pre-excitation which was associated with accessory pathway. The prevalence of WPW syndrome is $0.1 \sim 0.3 \%$ in general population [1]. Paroxysmal supraventricular tachycardia (PSVT) or atrial fibrillation (AF) is common arrhythmia in WPW syndrome. Using atrioventricular (AV) node blockers (adenosine, digoxin, non-dihydropyridine calcium channel blockers, and intravenous amiodarone) in patients with WPW syndrome who have pre-excited AF is potentially harmful because these treatments accelerate ventricular rate due to conduction of accessory pathway. So, radiofrequency catheter ablation (RFCA) of accessory pathway is recommended in symptomatic patients with pre-excited AF [2]. We report a case of pharmacological management of an elderly patient with PSVT and AF in WPW syndrome who could not undergo RFCA.

\section{Case Report}

An 80-year-old male patient admitted to emergency department due to ileus. He had undergone Whipple's operation thirteen years ago. Initial electrocardiography (ECG) showed normal sinus rhythm without delta wave. At hospital day 5 , his mental status became drowsy. Serum creatinine level increased and then anuria developed. He underwent continuous renal replacement therapy. After a while, urination recovered and PSVT occurred (Figure 1). Adenosine $6 \mathrm{mg}$ intravenous injection restored sinus rhythm without delta wave. At hospital day 6 , he complained chest pain and cardiac enzyme increased. Coronary angiography showed $50 \%$ stenosis at mid left anterior descending artery and distal left circumflex artery. During the coronary angiography, non-sustained AF was observed. On that evening, an irregular wide QRS complex tachycardia, which R-R interval was between $310 \mathrm{~ms}$ and $420 \mathrm{~ms}$, occurred and associated with hypotension and mental change (Figure 1). DC cardioversion restored sinus rhythm. ECG showed delta waves. The patients were diagnosed with WPW syndrome because of symptomatic PSVT or AF and delta waves during sinus rhythm. We considered electrophysiology (EP) study and RFCA of accessory pathway in WPW syndrome with PSVT and AF because 2015 

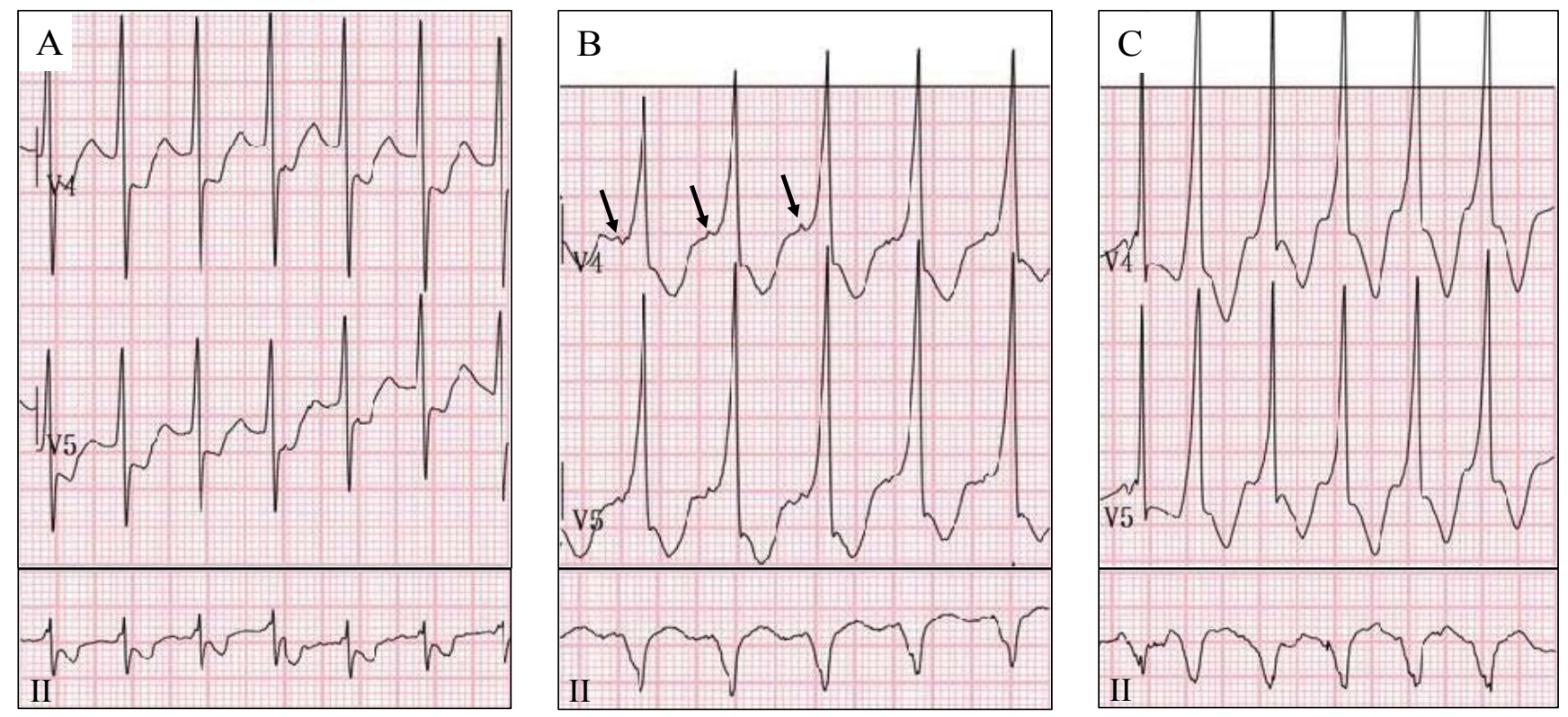

Figure 1: Electrocardiography. A) Orthodromic atrioventricular reentrant tachycardia showing regular tachycardia with narrow QRS complex; B) Antidromic atrioventricular reentrant tachycardia showing regular tachycardia with wide QRS complex. P waves are observed (arrow); C) Atrial fibrillation rapidly conducted by accessory pathway showing irregular tachycardia with wide QRS complex. P waves are not observed.
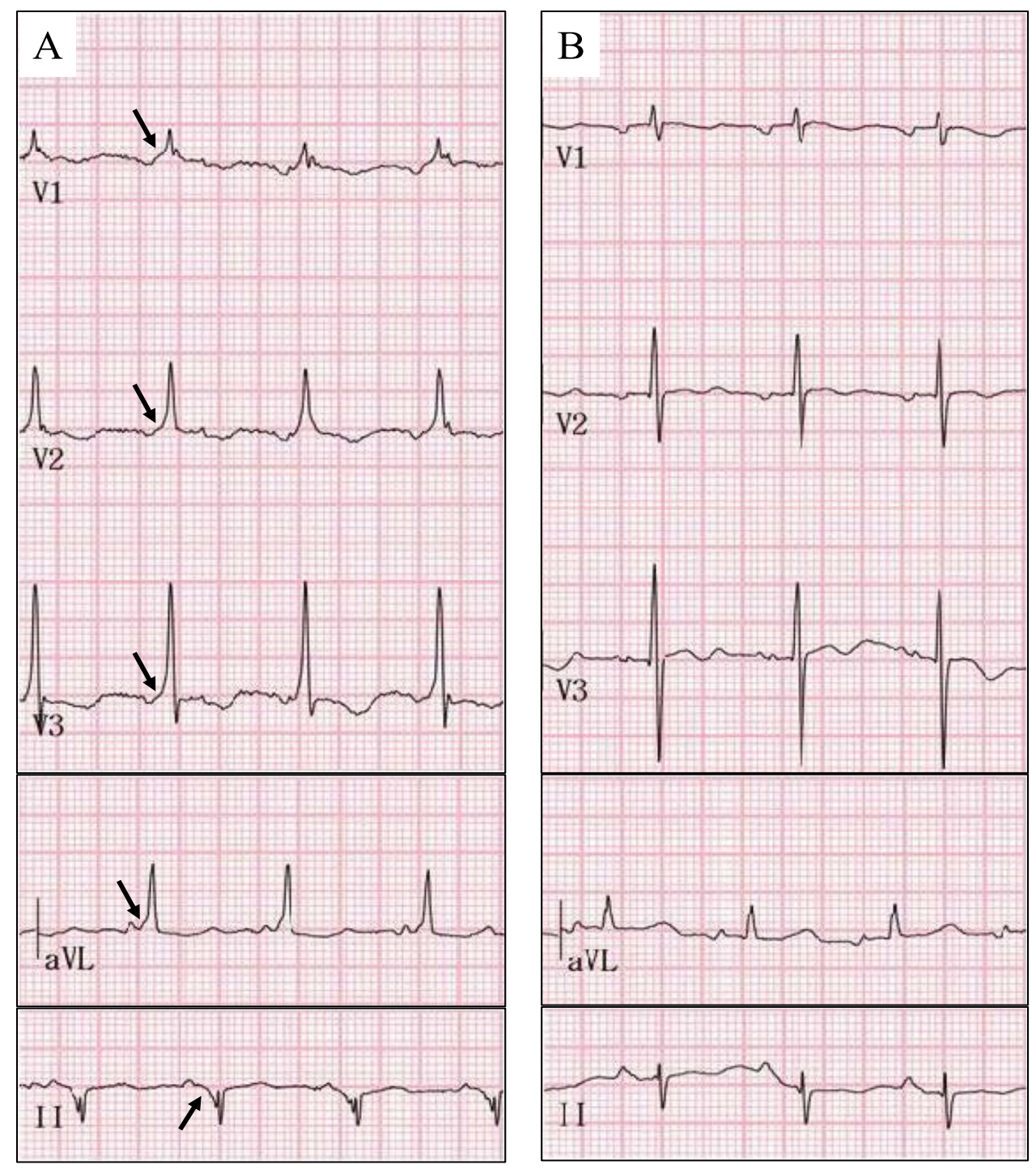

Figure 2: Electrocardiography. A) Delta waves (arrow) during sinus rhythm; B) Absence of delta waves after treatment with bisoprolol and flecainide. 
ACC/AHA/HRS SVT guideline recommends an EP study is useful in symptomatic patients with pre-excitation to risk-stratify to life-threatening arrhythmic events [3]. However, this elderly patient could not lie without moving in bed because of back pain and intermittent irritability from delirium. So, his family did not want the invasive procedure such as RFCA. We started bisoprolol $1.25 \mathrm{mg}$ twice a day and flecainide $75 \mathrm{mg}$ twice a day for AF without structural heart disease in consideration of old age and increased creatinine level $(1.57 \mathrm{mg} / \mathrm{dl})$. After that, one episode of PSVT occurred. Dosages of bisoprolol and flecainide were increased up to usual adult dosages as $2.5 \mathrm{mg}$ twice a day and $100 \mathrm{mg}$ twice a day, respectively. Thereafter, PSVT or AF has not occurred and delta waves have not appeared (Figure 2).

\section{Discussion}

The clinical significance of WPW syndrome is occurrence of PSVT and AF. The most common tachycardia in this syndrome is PSVT which is atrioventricular reentrant tachycardia (AVRT). The most patients with tachycardia in WPW syndrome have good prognosis [4]. Sudden cardiac death occurs rarely, with an estimated frequency of $0.1 \%$ [5]. AF in WPW syndrome is a potentially life-threatening arrhythmia. The incidence of paroxysmal AF has been reported to be $10 \sim 38 \%$ of patients with WPW syndrome [6]. ECG shows fast, irregular wide or narrow QRS complex tachycardia.

Treatment of patients with PSVT or AF in WPW syndrome depends on hemodynamic stability. Hemodynamically unstable patients should receive synchronized cardioversion immediately. In hemodynamically stable patients with orthodromic AVRT, adenosine is beneficial for acute treatment. Intravenous diltiazem, verapamil, or beta blockers can be effective for acute treatment in patients with orthodromic AVRT who do not have pre-excitation on their resting ECG during sinus rhythm [3]. In hemodynamically stable patients with pre-excited $A F$ and rapid ventricular response, intravenous procainamide or ibutilide to restore sinus rhythm or slow the ventricular rate is recommended [2].

In Korea, ibutilide or procainamide is not available. Class III anti-arrhythmic agents and AV node blockers should not be used for AF in WPW syndrome. Because, the block of AV node conduction can increase anterograde conduction of accessory pathway and lead to ventricular arrhythmia including ventricular tachycardia (VT) or fibrillation (VF) [7].

RFCA is recommended for the long term therapy of WPW syndrome with PSVT or AF. However, the recurrence rate of paroxysmal AF after successful RFCA of accessory pathway is about $55 \%$ in patients over 60 years of age [8]. Pharmacological therapy after successful ablation should be considered for maintaining sinus rhythm.

Flecainide increases the accessory pathway refracto- ry period and blocks anterograde conduction across the accessory pathway in $40 \%$ of cases. However, flecainide has a lesser effect on refractory period of atrioventricular node [9]. Flecainide also decreases the development of AF in the absence of structural heart disease. Amiodarone should be preferred in AF with structural heart disease [2]. Beta blocker is also considered in patients with arrhythmia related to an accessory pathway with a short refractory period. So, concomitant administration of beta blocker and flecainide results in greater long-term efficacy and can be one of options for the treatment of a patients with AF in WPW syndrome [9].

In our case, a patient with PSVT and AF in WPW syndrome has been treated with class Ic anti-arrhythmic drug, flecainide and class II anti-arrhythmic drug, bisoprolol. After then, PSVT or AF has not recurred for 1 month. This combination therapy is effective in the elderly patient who is unable to undergo RFCA. Delta waves disappear in ECG.

\section{Conclusion}

Co-administration of flecainide with bisoprolol is an effective management for PSVT or AF in an elderly patient with WPW syndrome who cannot be treated with RFCA. This pharmacological therapy may cause absence of delta waves.

\section{Conflict of Interest}

Authors declare no conflict of interest.

\section{References}

1. Sidhu J, Roberts R (2003) Genetic basis and pathogenesis of familial WPW syndrome. Indian Pacing Electrophysiol J 3: $197-201$

2. January CT, Wann LS, Alpert JS, Calkins H, Cigarroa JE, et al. (2014) 2014 AHA/ACC/HRS guideline for the management of patients with atrial fibrillation: A report of the american college of cardiology/american heart association task force on practice guidelines and the heart rhythm society. J Am Coll Cardiol 64: E1-E76.

3. Page RL, Joglar JA, Caldwell MA, Calkins $H$, Conti JB, et al. (2016) 2015 ACC/AHA/HRS guideline for the management of adult patients with supraventricular tachycardia: A report of the american college of cardiology/american heart association task force on clinical practice guidelines and the heart rhythm society. Circulation 133: E506-E574.

4. Munger TM, Packer DL, Hammill SC, Feldman BJ, Bailey $K R$, et al. (1993) A population study of the natural history of wolff-parkinson-white syndrome in Olmsted County, Minnesota, 1953-1989. Circulation 87: 866-873.

5. Zardini M, Yee R, Thakur RK, Klein GJ (1994) Risk of sudden arrhythmic death in the Wolff-Parkinson-White syndrome: Current perspectives. Pacing Clin Electrophysiol 17: $966-975$.

6. Asano Y, Kaneko K, Matsumoto K, Saito J, Yamamoto T, et al. (1991) Atrial fibrillation and atrial vulnerability in the wolff-parkinson-white syndrome. Jpn Circ J 55: 287-296.

7. Pietersen AH, Andersen ED, Sandoe E (1992) Atrial fibrillation in the Wolff-Parkinson-White syndrome. Am J Cardiol 70: 38-43. 
8. Dagres N, Clague JR, Lottkamp H, Hindricks G, Breithardt $G$, et al. (2001) Impact of radiofrequency catheter ablation of accessory pathways on the frequency of atrial fibrillation during long-term follow-up; high recurrence rate of atrial fibrillation in patients older than 50 years of age. Eur Heart J 22: 423-427.

9. Crozier I (1992) Flecainide in the wolff-parkinson-white syndrome. Am J Cardiol 70: 26-32. 